\title{
SIMULATION OF THE DYNAMICS OF NON- RESONANT MOTION IN A CONTROLLED DESCENT OF AN ASYMMETRIC SPACECRAFT IN THE LOW-DENSITY ATMOSPHERE
}

\author{
V.V. Lyubimov, E.V. Kurkina \\ Samara National Research University, Samara, Russia
}

\begin{abstract}
We consider the problem of implementation of non-resonant motion at descent of a spacecraft with small mass and aerodynamic asymmetries in the low-density atmosphere of Mars. A controllable decrease in the mass asymmetry contributes to implementation of non-resonant motion of the spacecraft. We introduce an analytical control law for the magnitude of asymmetry. The results of numerical simulation of spherical motion of a spacecraft with controllable asymmetry are included.
\end{abstract}

Keywords: spacecraft, resonance, control, atmosphere, asymmetry

Citation: Lyubimov VV, Kurkina EV. Simulation of the dynamics of nonresonant motion in a controlled descent of an asymmetric spacecraft in the lowdensity atmosphere. CEUR Workshop Proceedings, 2016; 1638: 610-621. DOI: 10.18287/1613-0073-2016-1638-610-621

\section{Introductions}

Resonance phenomena in uncontrolled descent in the atmosphere of a reentry spacecraft (SC) with small aerodynamic and mass asymmetry are discussed in a significant number of works: [1] - [3], and others. The issue of a controlled atmospheric motion of a reentry spacecraft in the vicinity of resonances was also explored in a significant amount of publications. For example, in [4] when changing the magnitude and direction of the displacement of the center of mass of the spacecraft during an asymmetric ballistic descent in the atmosphere, the problem of limitation of the angle of attack and angular velocity in motion control within a small neighborhood of the rotational resonance has been studied. As a small asymmetry, the following contributing factors have been taken into account: displacement of the center of mass and an asymmetry leading to deviation of the angle of attack. In [5] - [6], the resonant motion of a reentry spacecraft with invariable position of the center of mass in the Martian atmosphere influenced by biharmonic stabilizing moment has been studied. 
In [7] - [9] it was described that the mechanical moments resulting from aerodynamic and mass asymmetries can cause evolution of angular velocity of a spacecraft to the magnitudes where a long resonance occurs. In [7], [8] it has been shown that nonresonant evolution of angular velocity of an asymmetrical spacecraft in the vicinity of the resonance is a secondary resonance effect [10]. Secondary resonance effects and associated phenomena of external stability of resonances, concerning the problem of asymmetric disturbed rotational motion of a satellite in orbit were studied in [11] and [12], respectively. It is known that the achievement of the resonant values of angular velocity does not guarantee implementation of a long resonance, as the initial conditions at the separatrix, leading to capture and passage through the resonance, are mixed, and it is necessary to calculate the probability of capture or passage through the resonance. [13]. The phenomenon of capturing a spacecraft with a small asymmetry in the resonance, being a random event, can be analyzed using the method of statistical tests [14]. Long resonant mode of motion of a spacecraft leads to achievement of high magnitude of angle of attack that can cause emergency situations involving failure when deploying the parachute system. It is an important practical task to maintain the asymmetry at minimal level. However, in the design of actual reentry spacecraft it is usually impossible to achieve zero mass and aerodynamic asymmetries. For this reason, there is a problem on controlled reduction of asymmetry in the process of reentry of a spacecraft in the atmosphere. In particular, the study [15] considers the problem of control of non-resonant evolution at rotational motion of a spacecraft moving in the vicinity of the resonance. In [15] the small spacecraft had a constant mass and low variable aerodynamic asymmetry. In [15] it has been shown that control of the level of aerodynamic asymmetry allows decreasing angular acceleration and providing a non-resonant mode of motion for the entire time of descent.

\section{Problem Statement}

In the process of separating from the base orbiting space module, the reentry spacecraft receives some angular momentum of a small magnitude [16]. As the result, the values of angular velocity of the spacecraft have achieved $\omega_{x}(0), \omega_{y}(0), \omega_{z}(0)$, which should be considered as initial on descent of the spacecraft into the low-density atmosphere of Mars. The said angular velocities are given in the main body-fixed coordinate system OXYZ. The coordinate system origin $\mathrm{O}$ is the center of mass of the spacecraft. Let as assume that the spacecraft has a conical shape, combined with a spherical surface. Let the axis OX is the axis parallel to the axis of symmetry of the cone. At reentry, the spacecraft is oriented with cone part towards the pressure head. During the atmospheric descent, the spacecraft conducts a spherical motion relative to the center of mass. We study the case when the vectors of the mechanical moments of low mass and aerodynamic asymmetry of the spacecraft are collinear. It is known [7] that at a collinear arrangement of vectors of these asymmetries, no occurrence of secondary resonance effects would take place in the system. The resonance values of angular velocity at various aerodynamic parameters can be found by applying the method of integral manifolds [17]. The values of angular velocity $\omega_{x}$ corresponding 
to the resonance of a lower order, called the principal resonance, are defined as follows [7]: $\omega_{x}^{r}= \pm \omega / \sqrt{1-\bar{I}_{x}}$. Here $\omega=\sqrt{-m_{z p} q S L c t g \alpha / I}, m_{z p}$ are the coefficient of stabilizing moment for the angle of attack $\alpha, \mathrm{q}$ is the dynamic pressure, $\mathrm{S}$ is the area of the SC's section, $\mathrm{L}$ is the length of the spacecraft, $\bar{I}_{x}=I_{x} / I, I=\left(I_{y}+I_{z}\right) / 2$, $I_{x}, I_{y}, I_{z}$ are the principal central moments of inertia of the spacecraft. In [18] it has been shown that during the descent in the atmosphere with low angles of attack during the passage through the main resonance, perturbations of angle of attack are substantially higher than at multiple resonances. Let us suppose that at the spacecraft's descent in the Martian atmosphere a controlled decrease in the values of parameters of small mass asymmetry of the spacecraft is implemented, while maintaining the same values of the parameters of low aerodynamic asymmetry. At reducing the mass asymmetry, we will not take into account the influence of non-resonant rotation on possible occurrence of resonance. This assumption is appropriate, in particular, if the vectors of moments of mass and aerodynamic asymmetries remain collinear. The aim of this study is to provide non-resonant motion of the spacecraft with a small aerodynamic and mass asymmetries descending into the atmosphere of Mars. To achieve this objective we solve the problem of controlled decreasing of the value of mass asymmetry of a spacecraft that also has invariable small aerodynamic asymmetry. Decrease in the value of mass asymmetry reduces the probability of capture into the main resonance [9]. To solve the problem, it is necessary to select a control law for the value of mass asymmetry what would allow the system to provide a passage through the resonance with a probability close to 1 .

\section{Mathematical Models}

A nonlinear low-frequency system of equations of motion of a spacecraft with a small mass and aerodynamic asymmetry (which can be obtained through the method of integral manifolds [19]) has the following form [8]:

$$
\begin{aligned}
& \overline{\mathrm{I}}_{\mathrm{x}} \frac{\mathrm{d} \omega_{\mathrm{x}}}{\mathrm{dt}}=-\varepsilon \mathrm{m}_{\mathrm{x}}^{\mathrm{A}} \sin \left(\theta+\theta_{2}\right), \\
& \frac{\mathrm{d} \alpha}{\mathrm{dt}}=-\Psi \frac{\omega^{2} \operatorname{tg} \alpha}{4 \omega_{\mathrm{a}}^{2} \pi} \mp \varepsilon \frac{\mathrm{m}^{\mathrm{A}}}{2 \omega_{a}} \cos \left(\theta+\theta_{1}\right), \\
& \frac{\mathrm{d} \theta}{\mathrm{dt}}=\omega_{\mathrm{x}}-\omega_{1,2}, \\
& \frac{\mathrm{d} \omega}{\mathrm{dt}}=\varepsilon \frac{\omega}{2 \mathrm{q}} \frac{\mathrm{dq}}{\mathrm{dt}} .
\end{aligned}
$$

Here $\varepsilon$ is a small parameter, $\theta=\varphi-\pi / 2, \varphi$ is the aerodynamic roll angle, $m_{x}^{A}, m^{A}$ are functions that determine the value of a mass and aerodynamic asymmetries, 


$$
\begin{aligned}
& m^{A}=\sqrt{\left(m_{1}^{A}\right)^{2}+\left(m_{2}^{A}\right)^{2}}, m_{1}^{A}=-\frac{\left(1+\bar{I}_{x}\right) \omega_{x}-3 \omega_{1,2}}{2 \omega_{a}} \frac{\omega^{2}}{m_{z p}}\left(m_{y}^{f}-C_{x} \overline{\Delta z}\right) \operatorname{tg} \alpha- \\
& -\frac{\omega_{1,2} \omega^{2} \operatorname{tg}^{2} \alpha}{2 \omega_{a} m_{z p}}\left(C_{y n} \overline{\Delta z}\right), m_{2}^{A}=-\frac{\left(1+\bar{I}_{x}\right) \omega_{x}-3 \omega_{1,2}}{2 \omega_{a}} \frac{\omega^{2}}{m_{z p}}\left(m_{z}^{f}+C_{x} \overline{\Delta y}\right) \operatorname{tg} \alpha+
\end{aligned}
$$

$+\frac{\omega_{1,2} \omega^{2} \operatorname{tg}^{2} \alpha}{2 \omega_{a} m_{z p}}\left(C_{y n} \overline{\Delta y}\right), \theta_{1}, \theta_{2}$ are functions that determine the relative positions of the mass and aerodynamic asymmetries, $m_{x 1}^{A}=-\frac{\omega^{2}}{m_{z p}}\left(C_{y p} \overline{\Delta y}\right) \operatorname{tg} \alpha$, $m_{x 2}^{A}=-\frac{\omega^{2}}{m_{z p}}\left(C_{y p} \overline{\Delta z}\right) \operatorname{tg} \alpha, \quad \sin \theta_{1}=m_{1}^{A} / m^{A}, \cos \theta_{1}=-m_{2}^{A} / m^{A}, \sin \theta_{2}=-m_{x 1}^{A} / m_{x}^{A}$, $\cos \theta_{2}=m_{x 2}^{A} / m_{x}^{A}, C_{x}, C_{y p}$ are aerodynamic coefficients of drag and lifting forces, $m_{y}^{f}, m_{z}^{\phi}$ are small aerodynamic coefficients of the spacecraft shape asymmetry, $\overline{\Delta y}, \overline{\Delta z}$ are small displacements of the center of mass related to the length of the spacecraft. The generalized parameter of mass asymmetry $\bar{m}_{x}^{A}$ is calculated as follows:

$$
m_{x}^{A}=\sqrt{\left(m_{x 1}^{A}\right)^{2}+\left(m_{x 2}^{A}\right)^{2}} .
$$

In the second term on the right side of the second equation (1) the top sign should be selected in the case of $\omega_{x}>0$. Bottom signs in these terms are selected at $\omega_{x}<0$. In a numerical simulation of spherical movement of a reentry spacecraft we consider the system of equations (1) together with three differential equations for slowly varying parameters of the center of mass: angle of inclination of the trajectory $\vartheta(t)$, airspeed $\mathrm{V}(\mathrm{t})$, altitude $\mathrm{H}(\mathrm{t})$.

We introduce the notation for the resonant frequency ratio $\Delta=\omega_{\mathrm{x}}-\omega_{1,2}$. The full derivative of the function $\Delta(t)$, calculated taking into account of (1) is equal to: $\frac{d \Delta}{d t}=P\left(\omega_{x}, \alpha, \omega\right)=\frac{\partial \Delta}{\partial \omega_{x}} \frac{d \omega_{x}}{d t}+\frac{\partial \Delta}{\partial \alpha} \frac{d \alpha}{d t}+\frac{\partial \Delta}{\partial \omega} \frac{d \omega}{d t}$. We perform the replacement of variables: $\tau=\sqrt{\varepsilon} t, \mu=\sqrt{\varepsilon}, \rho=\Delta / \sqrt{\varepsilon}$. As a result, the system (1) is written in the "pendulum" form:

$$
\begin{aligned}
& \frac{\mathrm{d} \sigma}{\mathrm{d} \tau}=\mu E(\sigma, \mu \rho, \theta, \mu), \\
& \frac{\mathrm{d} \rho}{\mathrm{d} \tau}=P(\sigma, \mu \rho, \theta, \mu),
\end{aligned}
$$


$\frac{\mathrm{d} \theta}{\mathrm{d} \tau}=\rho$,

where $\sigma=(\alpha, \omega), E=\frac{d \rho}{d \tau}$. Here the functions $\mathrm{E}$ and $\mathrm{P}$ are periodically in phase $\theta$ with the period $2 \pi$. Consider the possibility of the existence of resonant modes of motion, corresponding to the equality $\Delta=0$. When $\mu=0$, then the system of equations (2) takes the form of the unperturbed system:

$\frac{\mathrm{d} \rho_{0}}{\mathrm{~d} \tau}=P\left(\sigma_{0}, \theta_{0}\right)$,

$\frac{\mathrm{d} \theta_{0}}{\mathrm{~d} \tau}=\rho_{0}$.

Here $\sigma_{0}=$ const. Movement in the vicinity of the resonance $\Delta=0$ is performed with a small perturbation of the system (4). The trivial solution of the unperturbed system (4) has the form:

$$
\begin{aligned}
& P\left(\sigma_{0}, \theta_{0}^{*}\right)=0, \\
& \rho_{0}^{*}=0 .
\end{aligned}
$$

Here $\theta_{0}^{*}, \rho_{0}^{*}$ is unperturbed values of the variables $\theta_{0}, \rho_{0}$, the corresponding resonance $\Delta=0$. From the solution of the first equation (5) we can find the magnitude of the phase $\theta_{0}^{*}$, in which the possible realization of the resonance $\Delta=0$.

Given $\frac{d \Delta}{d t}=P\left(\omega_{x}, \alpha, \omega\right)$, we write the equation $P\left(\omega_{x}, \alpha, \omega\right)=0$ in the following form:

$$
\sin \left(\theta+\theta_{3}\right)=\frac{f\left(\omega_{x}, \alpha, \omega\right)}{m_{f}\left(\omega_{x}, \alpha, \omega\right)} .
$$

Here $f\left(\omega_{x}, \alpha, \omega\right)=\left|\frac{\partial \Delta}{\partial \alpha}\left(-\frac{\omega^{2} \Psi \operatorname{tg} \alpha}{\omega_{a} F_{a} \pi}\right)+\frac{\partial \Delta}{\partial \omega} \frac{d \omega}{d t}\right|, m_{f}=\sqrt{\left(m_{1}^{f}\right)^{2}+\left(m_{2}^{f}\right)^{2}}$,

$$
\begin{aligned}
& m_{1}^{f}= \pm \frac{\partial \Delta}{\partial \alpha}\left(\frac{2 \bar{m}^{A} \omega^{2}}{F_{a}}\right) \sin \theta_{1}+\frac{\partial \Delta}{\partial \omega_{x}}\left(-\frac{\bar{m}_{x}^{A} \omega^{2}}{\bar{I}_{x}}\right) \cos \theta_{2}, \bar{m}^{A}=m^{A} / \omega^{2}, \\
& \bar{m}_{x}^{A}=m_{x}^{A} / \omega^{2}, m_{2}^{f}=\mp \frac{\partial \Delta}{\partial \alpha}\left(\frac{2 \bar{m}^{A} \omega^{2}}{F_{a}}\right) \cos \theta_{1}+\frac{\partial \Delta}{\partial \omega_{x}}\left(-\frac{\bar{m}_{x}^{A} \omega^{2}}{\bar{I}_{x}}\right) \sin \theta_{2} .
\end{aligned}
$$

Therefore, in the unperturbed case, the equation $\left|\sin \left(\theta_{0}^{*}+\theta_{3}\right)\right|=1$ takes the form:

$$
f\left(\omega_{x}, \alpha, \omega\right)=m_{f}\left(\omega_{x}, \alpha, \omega\right) .
$$


From the solution of equation (7) can be used to find minimal values of the asymmetry parameters of the spacecraft, in which the resonance $\Delta=0$ is realized. We will denote these values of the asymmetry parameters as: $\bar{m}_{x}^{A k}, \bar{m}^{A k}$.

\section{Research of Dynamics and Control of Spacecraft Motion}

Let the angular velocity ${ }^{\omega_{x}}$ change from some initial positive value according to the first equation of the system (1) to the resonance values $\omega_{x}^{r}$. In addition, we assume that during the descent of the spacecraft the ratio $\theta_{1}-\theta_{2}=3 \pi / 2$ remains unchanged, which corresponds to the collinear arrangement of vectors of moments resulting from mass and aerodynamic asymmetries.

The value of the asymmetry parameter $\bar{m}_{x}^{A}=m_{x}^{A} / \omega^{2}$ under which the passage through the main resonance at small angles of attack is guaranteed, must satisfy the condition: $\bar{m}_{x}^{A} \leq m_{x}^{A k}=\frac{\bar{I}_{x}}{\sqrt{1-\bar{I}_{x}}} \frac{|d \omega / d t|}{\alpha \omega^{2}}$ [9]. In another form, this condition can be written as: $\Delta m_{x}^{A} \leq 0$, where $\Delta m_{x}^{A}=\bar{m}_{x}^{A}-\bar{m}_{x}^{k}$. When calculating the expression contained in the right part of this condition it is necessary to make the calculation of values $\alpha, \omega, d \omega / d t$ on the separatrix that divides the resonance area of the oscillations and the area of non-resonant rotation [20].

We introduce the following control law for the parameter of mass asymmetry:

$\bar{m}_{x}^{A}=k_{1} \exp \left(k_{2} H(t)\right) \sin \left(k_{3} H(t)\right)$,

where $k_{1}, k_{2}, k_{3}$ are positive small constant control coefficients.

The control law (8) is an analytical dependence of the parameter of mass asymmetry $\bar{m}_{x}^{A}$ of flight altitude $\mathrm{H}$. Therefore, we can apply properties of analytic functions to this law [21]. During the descent in the atmosphere, the flight altitude $\mathrm{H}$ is decreasing. As the result (in accordance with the control law (8)), the value of the parameter mass asymmetry $\bar{m}_{x}^{A}$ is decreasing. If during the descent in the atmosphere the decreasing of the parameter of asymmetry $\bar{m}_{x}^{A}$ reaches a value at which the condition $\bar{m}_{x}^{A} \leq m_{x}^{A k}$ is fulfilled, then at small angles of attack $\alpha$ a guaranteed passage through the main resonance occurs.

Fig.1 is a functional block diagram of a control system for the parameter of mass asymmetry $\bar{m}_{x}^{A}(t)$. In this control system, only one control channel is provided: angular velocity $\omega_{x}$. During operation of the said control system, a decrease in mass asymmetry parameter $\bar{m}_{x}^{A}(t)$ is achieved, ensuring fulfillment of the conditions 
$\bar{m}_{x}^{A} \leq m_{x}^{A k}$. As a result, there is a guaranteed passage of the system through the main resonance. In the following, there is no control of the value $\bar{m}_{x}^{A}(t)$ in accordance with the law (8) and the parameter $\bar{m}_{x}^{A}(H)$ is calculated according to expression (2). This control system is based on the assumption that at spherical movement at atmospheric of a spacecraft a non-resonant evolution of the angular velocity caused by occurrence of the secondary resonance effects would not occur. Therefore, the control system is operable, for example, on condition of $m^{A}=0$ or in the case of a special form of asymmetry $\left(\theta_{1}-\theta_{2}=\frac{\pi}{2}, \frac{3 \pi}{2}\right)$.

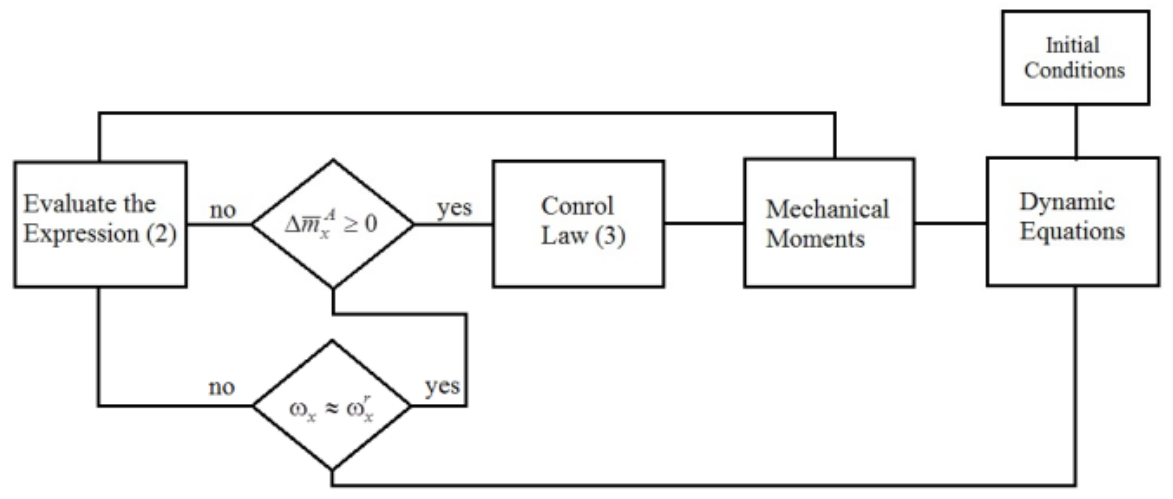

Fig. 1. Functional block diagram of a control system for the parameter of asymmetry $\bar{m}_{x}^{A}(t)$

\section{Numerical results}

In numerical simulation of evolution of the angular velocity $\omega_{x}(t)$ we use the mathematical model of motion of the spacecraft, including: the system of equations (1), three differential equations of motion for changing the center of mass of the reentry spacecraft [2] and the control law (8). Equation (7) defines a three-dimensional space $\left(\bar{m}_{x}^{A}, \bar{m}^{A}, f / \omega^{2}\right)$ the surface of critical values of the asymmetry parameters $\bar{m}_{x}^{A k}$, $\bar{m}^{A k}$, shown in Fig.2.

It is possible to show that the points of this space, lying below the surface provide a guaranteed passage of the system through resonance $\Delta=0$. If the points belong to that surface or are above it, then the probability of capture into resonance is not zero in cases $\theta_{1}-\theta_{2} \neq \frac{\pi}{2}, \frac{3 \pi}{2}$. 


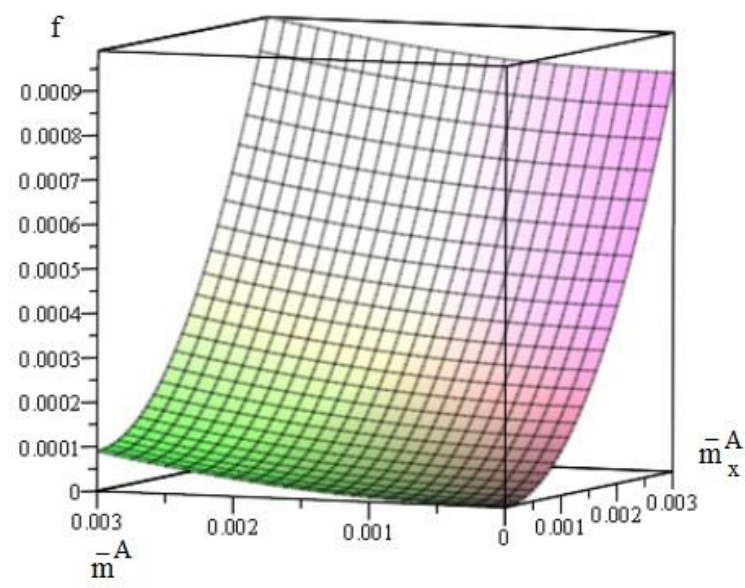

Fig. 2. The surface of critical values of asymmetry parameters

Some representative results of numerical simulation in case of small angle of attack are shown in Fig. 3-6. Fig. 3 and Fig. 5 show the variation of the asymmetry parameter $\bar{m}_{x}^{A}(t)$ and the critical values of the parameter $\bar{m}_{x}^{A}(t)$ defined in case of small angles of attack. Fig. 4 and Fig. 6 show the angular velocity $\omega_{x}(t)$ and resonance values $\omega_{x}^{r}(t)$. The initial value of the angular velocity $\omega_{x}(0)$ in drawing up the Fig. 4 and Fig. 6 had a value greater than $\omega_{x}^{r}(0)$ and was equal to $0.8 \mathrm{rad} / \mathrm{s}$. The numerical results show that up to angles of attack of $0.6 \mathrm{rad}$ the value $m_{x}^{A k}=\frac{\bar{I}_{x}}{\sqrt{1-\bar{I}_{x}}} \frac{|d \omega / d t|}{\alpha \omega^{2}}$ is virtually equal to the corresponding critical parameter of the asymmetry obtained for arbitrary angles of attack. Numerical results of control of the value of angular velocity $\omega_{x}(t)$ are determined by the choice of the coefficients $k_{1}, k_{2}, k_{3}$. Fig. 3 shows a case where the initial values of $\bar{m}_{x}^{A}(0)>\bar{m}_{x}^{A k}(0)$. However, controlled decrease of the parameter $\bar{m}_{x}^{A}(t)$ provides for achievement of values $\bar{m}_{x}^{A}(t)$ smaller than the current values $\bar{m}_{x}^{A k}$. Fig. 4 indicates that the said decrease in $\bar{m}_{x}^{A}(t)$ is accompanied by a gradual decrease in the frequency of resonance oscillations, followed by leaving the resonance.

Fig. 5-6 shows the results corresponding to another case. Here the choice of parameters $k_{1}, k_{2}, k_{3}$ contributes to the following conditions: $\bar{m}_{x}^{A}(0)<\bar{m}_{x}^{A k}(0)$. In this case, at controlled reduction of the parameter $\bar{m}_{x}^{A}(t)$, the condition $\bar{m}_{x}^{A}(t)<\bar{m}_{x}^{A k}(t)$ remains true within the entire time of descent of the spacecraft in the atmosphere. This is shown in Fig. 5. The results in Fig. 6 correspond to the variation of the angular 
velocity shown in Fig. 5. Here, the small value of the parameter $\bar{m}_{x}^{A}(t)$ contributes to implementation of two passages of the system through the main resonance as observed at the intersection of the curves $\omega_{x}(t)$ and $\omega_{x}^{r}(t)$.

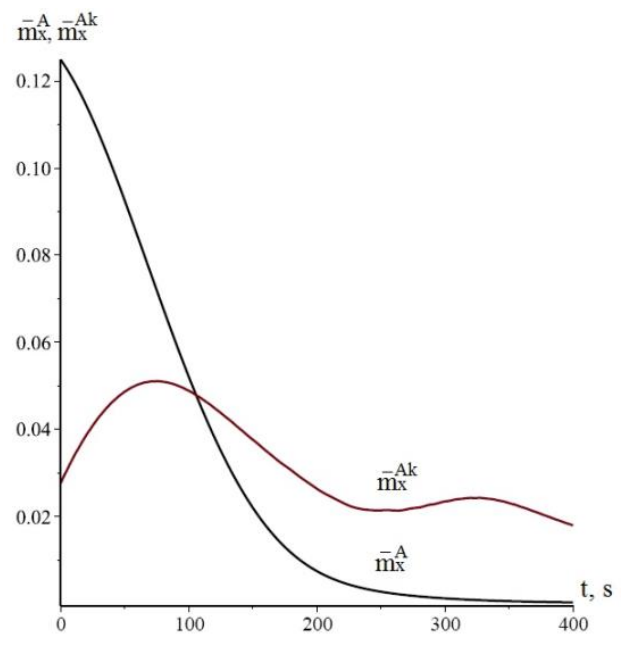

Fig. 3. Reducing the value of the asymmetry parameter $\bar{m}_{x}^{A}(t)$ when $\bar{m}^{A}=0.003$,

$$
\bar{m}_{x}^{A}=0.001 \exp \left(5 \cdot 10^{-4} \cdot H(t)\right) \sin \left(10^{-5} \cdot H(t)\right)
$$

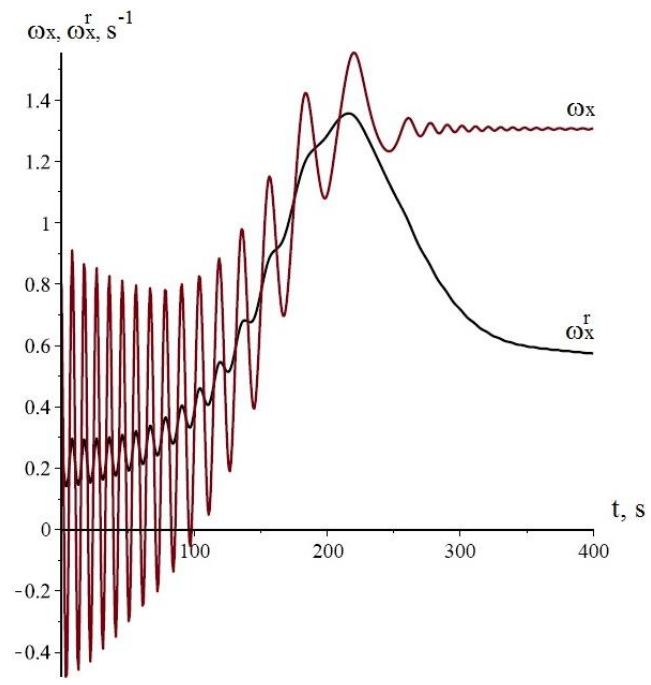

Fig. 4. Long-term resonance changing into a non-resonant motion in the case of $\bar{m}=0.003$,

$$
\bar{m}_{x}^{A}=0.001 \exp \left(5 \cdot 10^{-4} \cdot H(t)\right) \sin \left(10^{-5} \cdot H(t)\right)
$$


Numerical results shown in Fig. 3-6 describe the process of continuous control of rotational motion of a reentry spacecraft based on the use of the law of reduction of the mass asymmetry parameter (3). In this sense, these results do not fully correspond to the operation of the control system shown in Fig. 1. These numerical results are not to describe the operation of a control system, but to demonstrate the important aspects of the behavior of angular velocity from a theoretical point of view. In addition, we consider the case where the control of the value of the parameter of mass asymmetry $\bar{m}_{x}^{A}(t)$ is implemented under the following constant values of generalized asymmetry parameters: $\theta_{1}-\theta_{2}=\pi$ and $\bar{m}_{x}^{A}(t)=0.003$. It is known that at the value $\theta_{1}-\theta_{2}=\pi$ in the systems equations of motion of a spacecraft it is possible for non-resonant evolution to occur, $\omega_{x}(t)$ caused by the secondary resonance effects. However, in Fig. 3 spherical movement begins from resonant oscillations, and the subsequent movement is not accompanied by non-resonant evolution of $\omega_{x}(t)$ as small values $\bar{m}_{x}^{A}(t)$ are achieved (in the non-resonant area). The results of numerical simulation displayed in Fig. 6 also show that there is no distinctive non-resonant evolution of $\omega_{x}(t)$ associated with occurrence of secondary resonance effects during the fulfillment of the condition $\bar{m}_{x}^{A}(t) \ll \bar{m}_{x}^{A k}(t)$. Therefore, in the cases considered in the numerical simulation, the control system shown in Fig.1 can be used for implementation of nonresonant descent of spacecraft in the low-density Martian atmosphere.

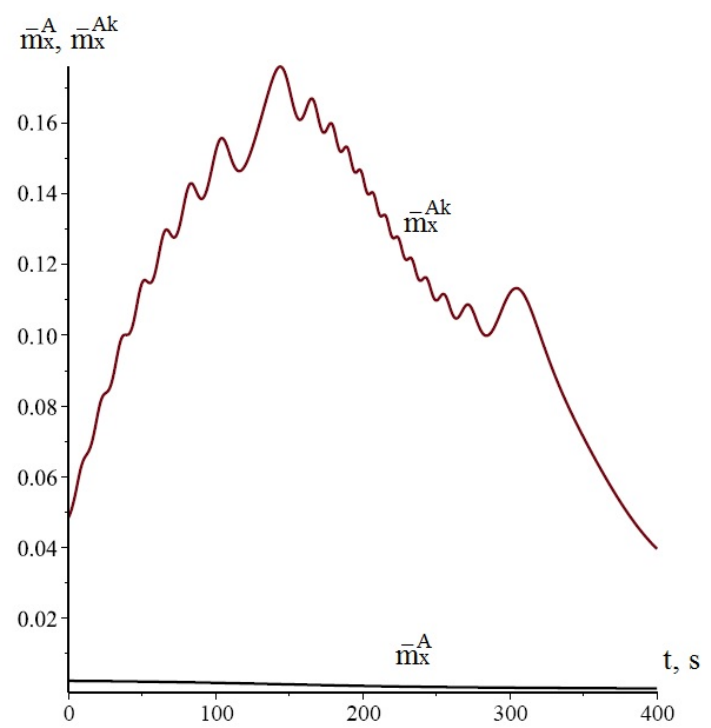

Fig. 5. Reducing the value of the asymmetry parameter $\bar{m}_{x}^{A}(t)$ when $\bar{m}^{A}=0.0015$,

$$
\bar{m}_{x}^{A}=0.001 \exp \left(10^{-5} H(t)\right) \sin \left(10^{-5} H(t)\right)
$$




\section{Conclusion}

In this study, spherical controlled movement of a reentry spacecraft was considered without taking into account non-resonant evolutions of angular velocity caused by, for example, the influence of the secondary resonance effects. Control of angular motion of a spacecraft providing non-resonant motion of the spacecraft based on the evolution of non-resonant angular velocity $\omega_{x}(t)$ is beyond this study. However, this issue can be explored in the following studies.

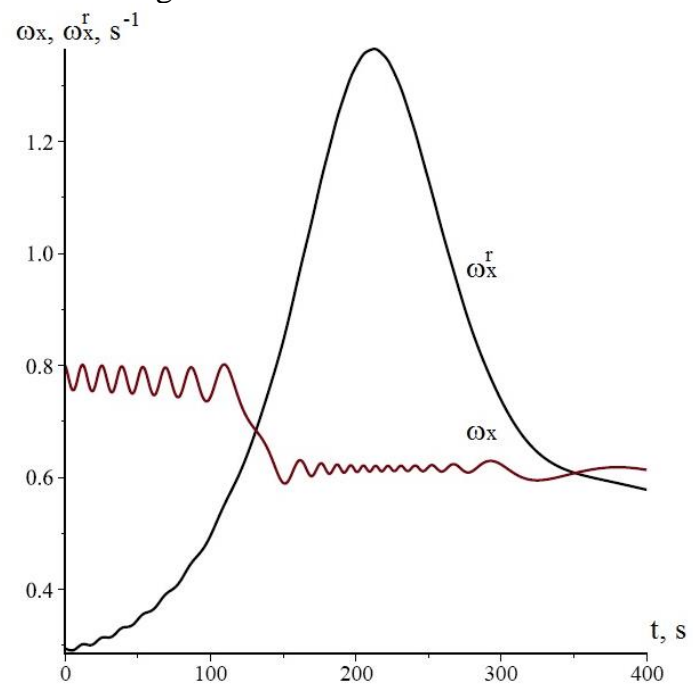

Fig. 6. Double passage through the resonance when $\bar{m}^{A}=0.0015$,

$$
\bar{m}_{x}^{A}=0.001 \exp \left(10^{-5} H(t)\right) \sin \left(10^{-5} H(t)\right)
$$

From the standpoint of practice, the problem of controlled reduction of the angle of attack at descent of a spacecraft with a small asymmetry in low-density atmosphere is also of immediate interest. To solve this problem, in addition to taking into account the damping aerodynamic moments, it is possible to investigate the influence of variable asymmetry at limiting values of the angle of attack.

\section{References}

1. Shilov AA, Goman MG. Resonant Modes of Spatial Uncontrolled Movement of a Spacecraft at Entry to the Atmosphere. Proceedings of TsAGI, 1975; 1624: 44. [in Russian]

2. Yaroshevsky VA. The movement of the body in an uncontrolled atmosphere. Moscow: Mechanical engineering, 1978; 168. [in Russian]

3. Aslanov VS. Nonlinear resonances in uncontrolled descent of asymmetric spacecrafts in the atmosphere. Cosmic Research, 1992; 30(5): 608-614. 
4. Platus DH. Roll Resonance Control of Angle of Attack for Re-entry Vehicle Drag Modulation. Journal of Guidance, Control, and Dynamics, 1981; 4(5): 632-636.

5. Aslanov VS, Ledkov AS. Features of Rotational motion of a spacecraft in the Martian atmosphere. Cosmic Research, 2007; 45(5): 331-338.

6. Aslanov VS. Resonance at motion of a body in the Mars's atmosphere under biharmonic moment. WSEAS Transactions on Systems and Control, 2008; 3(1): 33-39.

7. Zabolotnov YuM, Lyubimov VV. Secondary resonance effect in the motion of a spacecraft in the atmosphere. Cosmic Research, 1998; 36(2): 194-201.

8. Lyubimov VV. Asymptotic analysis of the secondary resonance effects in the rotation of a spacecraft with a small asymmetry in the atmosphere. Russian Aeronautics, 2014; 57(3): 245-252.

9. Lyubimov VV. External stability of resonances in dynamics of flight of a spacecraft with a small asymmetry. Publishing House of the Samara Scientific Center of the Russian Academy of Sciences, 2013; 276. [in Russian]

10. Sadov YuA. Secondary Resonance Effects in Mechanical Systems. Mechanics of Solids, 1990; 4: 20-24.

11. Lyubimov VV. Some features of disturbed rotational motion of a satellite with a strong onboard magnet. Russian Aeronautics, 2009; 52(2): 172-175.

12. Lyubimov VV. External stability of resonances in the motion of an asymmetric rigid body with a strong magnet in the geomagnetic field. Mechanics of Solids, 2010; 45(1): 10-21.

13. Neyshtadt AI. Capture into Resonance and Scattering on Resonances in Two-Frequency Systems. Differential equations and dynamical systems, Collection of articles, Proceedings of V.A. Steklov Mathematical Institute, Nauka, 2005: 198-218.

14. Zabolotnov YuM. Statistical Analysis of Movement of Light Capsule around of the Centre of Mass at Reentry into Atmosphere. Cosmic Research, 2013; 51(2): 1-12.

15. Lyubimov VV. Dynamics and Control of Angular Acceleration of a Reentry Spacecraft with a Small Asymmetry in the Atmosphere in the Presence of the Secondary Resonance Effect. 2015 International Siberian Conference on Control and Communications (SIBCON), 2015: 1-4.

16. Kalaev MP, Lyubimov VV, Semkin ND. Hardware-in-the-Loop and Simulation Modelling for a Process of Separation of a Microsatellite. Gyroscopy and Navigation, 2014; 85(2): 52-60

17. Zabolotnov YuM, Lyubimov VV. Application of the method of integral manifolds for construction of resonant curves for the problem of spacecraft entry into the atmosphere. Cosmic Research, 2003; 41(5): 453-459.

18. Zabolotnov YuM. Asymptotic analysis of quasi-linear equations of motion of a spacecraft with a small asymmetry in the atmosphere II Cosmic Research, 1994; 32(2): 22-33.

19. Strygin VV, Sobolev VA. Separation of Motions by the Integral Manifolds Method. Moscow: Nauka, 1988. [in Russian]

20. Bobylev AV, Yaroshevskiy VA. Assessment of conditions of capture of an uncontrolled body into a resonance rotating mode during descent into the atmosphere. Cosmic Research, 1999; 37(5): 512-524.

21. Korn G, Korn T. Mathematical Handbook for Scientists and Engineers. Moscow, Nauka, 1974: 832. 